\title{
Reservoirs of CTX-M extended spectrum $\beta$-lactamase-producing Enterobacteriaceae in Oaxaca, Mexico
}

\begin{abstract}
Objectives: To investigate putative reservoirs of CTX-M extended spectrum $\beta$-lactamaseproducing Enterobacteriaceae (ESBL-E) in Oaxaca, Mexico.

Methods: Samples from different putative reservoirs of these organisms were analyzed and included human and dog feces, tap water, fresh beef and chicken meat, salsas and vegetables from street vendors and domestic flies in household kitchens and restaurants. Identification of ESBL-E was performed according to CLSI guidelines; molecular characterization of bla $a_{\text {CTX-M }}$ strains was performed using PCR.

Results: Except for tap water, all the analyzed niches act as reservoir of ESBL-E clones in our community. The prevalence of either ESBL-E contamination or colonization varies depending on the reservoir under study, from very high (78/104) in human fecal carriage to non-existent $(0 / 30)$ in tap water; $96.2 \%(150 / 156)$ of the ESBL-E positive clones harbored the $b l a_{\text {СТХ-м }}$ gene.

Conclusions: Contamination or fecal colonization of ESBL-E clones in our community is largely spread and our results suggest that, if overuse of antibiotics plays a key role in the appearance of antibacterial resistance clones, the lack of hygiene measures may play a pivotal role in the spread of ESBL-E to humans.
\end{abstract}

Keywords: ESBL, antibiotic resistance, CTX-M, animal reservoirs

\section{Introduction}

In the last decades, antimicrobial resistance has become a global threat to public health systems in the world. Within the wide range of resistance mechanisms developed by bacteria, one of the most prevalent within the Gram negative organisms is the production of extended-spectrum $\beta$-lactamases (ESBL). These enzymes confer the producing microorganisms, most importantly $E$. coli and Klebsiella $s p$, resistance to most beta-lactam antibiotics, including penicillins, first-, second-, and third-generation cephalosporins, and the monobactam aztreonam; however, they remain susceptible to $\beta$-lactamase inhibitors, such as clavulanic acid. ${ }^{1}$

In the past, infections caused by ESBL-producing bacteria were limited to those associated with health care and by microorganisms carrying the TEM and SHV genes. However, since the description in Europe in the late 1990s of ESBL strains causing communityacquired infections (CAI), the number of cases reported worldwide has increased, and Mexico is no exception. ${ }^{2,3}$ This increase in the number of cases of CAI coincides with the increase in the number of isolates of CTX-M type strains, mainly of the CTX-M-15 subtype, which is currently the most frequently isolated strain in different parts of the world. As the number of CAI infections caused by these microorganisms is on the rise, so is the prevalence of health care-associated infections caused by ESBL-producing bacteria, as indicated globally by different authors. ${ }^{3-5}$ Additionally, hospital stay is a major risk of fecal contamination by these multi-resistant bacteria. ${ }^{4}$

Different bacterial infections, such as sepsis and those of the urinary tract (UTI) caused by members of the Enterobacteriaceae family have been suggested to have a gut origin..$^{67}$ Ruppé et al., ${ }^{8}$ showed that a high fecal abundance of ESBL-producing strains, appear to be associated with a high risk of developing UTI by these organisms;
Volume 7 Issue I - 2019

\author{
Mario Galindo-Méndez',2 \\ 'Laboratorios Galindo SC, Mexico \\ ${ }^{2}$ Universidad Anáhuac Oaxaca, Mexico
}

Correspondence: Mario Galindo-Méndez, Universidad Anáhuac Oaxaca, Blvd Guadalupe Hinojosa de Murat I 100, San Raymundo Jalpam, Oaxaca, 71248, Mexico, Tel +529515161303, Email magalindom@hotmail.com

Received: January 25, 2019 | Published: February 06, 2019 thus, fecal colonization by these strains seems to be an important first step in the pathogenesis of these infections. An additional factor in the pathogenesis of UTI, related to ESBL-producing bacteria, is the ability of these organisms to produce biofilm; ability found to be significantly higher in ESBL positive strains of uropathogenic E. coli than that of ESBL negative strains. ${ }^{9}$ Biofilm reduces antibiotic effectiveness as it halts the accumulation of the antibiotic to an effective concentration. ${ }^{10}$

Bacterial transmission of ESBL- producing Enterobacteriaceae (ESBL-E) to humans from different sources is well documented but varies from region to region, and they include, but are not limited to, fecal transmission from other people and animals ${ }^{11,12}$ and ingestion of contaminated food and water. ${ }^{13}$ The increasing threat of antibiotic resistance has prompted surveillance of drug-resistant bacteria from different reservoirs as a tool to understand their epidemiology and to implement infection control measures. The objective of this study was to investigate putative reservoirs of ESBL-E in the city of Oaxaca, Mexico.

\section{Ethics statement}

The study was approved by Oaxaca Site Management Organization SC ethics committee (COFEPRIS 17 CI 20067 150) with reference number CEI-OSMO 189/2018.

\section{Methods}

The study was performed in the southern Mexican city of Oaxaca from January to June 2018.

\section{Sampling}

To investigate putative reservoirs of ESBL-E the following niches were included in the study: 
a) Human feces. 104 adults who agreed to participate and submitted a fecal sample obtained through self-applied rectal swabs were included in the study; $59.6 \%$ of subjects were women $(n=62)$ and $40.4 \%$ were men $(n=42)$. Age of subjects ranged from 18 to 65 years.

b) Dog feces. 30 non-duplicate rectal swabs from companion dogs were taken. Age of dogs ranged from one to 8 years, including different breeds. Neither human subjects nor companion dogs had clinical symptoms and had not taken antibiotics nor had been hospitalized in the previous 3 months before stool collection. Samples were transported using commercial Stuart transport media (Transystem, USA).

c) Tap water. Thirty $200 \mathrm{~mL}$ water samples were collected using sterile glass bottles from households $(50 \%)$ and restaurants (50\%). All samples were collected from kitchen faucets.

d) Salsas/vegetables from street vendors. 84 non-duplicate samples were collected from local street vendors selling their food products. $50.0 \%(\mathrm{n}=42)$ of the samples were different types of salsas and $50.0 \%(\mathrm{n}=42)$, a mix of vegetables consisting of minced onions, parsley and lettuce or cabbage. All samples were ready-to-eat food.

e) Meat. A total of 50 uncooked samples of beef $(64 \%, n=32)$ or chicken meat $(36 \%, n=18)$ were provided by different local restaurants and households.

f) Flies. 120 common house flies (Musca domestica) were collected in kitchens from different households and restaurants using nontoxic sticky flypaper and harvested within $24 \mathrm{~h}$ after placement. All flies were analyzed in 24 separate pools of 5 flies, collected in each location, in each pool.

g) All samples were submitted to a private microbiology laboratory (Laboratorios Galindo SC, Oaxaca, Oaxaca), where samples were processed within two hours of collection. Written consent was obtained from all human subjects, street vendors and pet, household and restaurant owners.

\section{Bacteriological analysis}

a) Human and dog feces. Swabs were directly inoculated on CHROMID ESBL (bioMérieux, France) agar and incubated at $35 \pm 2{ }^{\circ} \mathrm{C}$ for $16-18$ hours.

b) Tap water. $100 \mathrm{~mL}$ of each sample were filtered through $0.45-\mu \mathrm{m}$ pore size filters (Sartorius, Goettingen, Germany) that were then transferred to CHROMID ESBL (bioMérieux, France) plates.

c) Salsas/vegetables/retail meat. $25 \mathrm{~g}$ of each sample were placed in a sterile stomacher bag with $100 \mathrm{~mL}$ of $0.1 \%$ peptone water. The stomacher bag was sealed and kneaded in a stomacher at $150 \mathrm{rpm}$ for $20 \mathrm{~min}$. The resulting wash was then serially diluted 4 logs in $0.1 \%$ peptone water and $100 \mu \mathrm{l}$ of $\log$ dilution were plated on CHROMID ESBL (bioMérieux, France) plates.

d) Flies. Fly pools were transferred to sterile bags containing phosphate-buffered saline, where they were thoroughly crushed using thumb and forefinger (from the outside of the bag) and then homogenized using a Stomacher400 (Seward, Worthing, United Kingdom) at 230rpm. Of these homogenates, $100 \mu 1$ was streaked on CHROMID ESBL medium (bioMérieux, France)

All plates were incubated at $35 \pm 2^{\circ} \mathrm{C}$ for $16-18$ hours. Possible ESBL-positive bacteria were confirmed using the Clinical Laboratory
Standard Institute (CLSI) phenotypic confirmatory test with

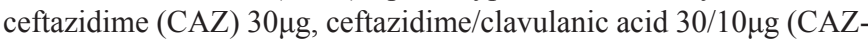
CLA), Cefotaxime (CTX) 30 $\mathrm{g}$, and Cefotaxime/clavulanic acid disks $30 / 10 \mu \mathrm{g} .{ }^{14}$ ESBL-producing isolates were defined as strains showing a " $\geq 5-\mathrm{mm}$ increase in a zone diameter for either antimicrobial agent tested in combination with clavulanate versus the zone diameter of the agent when tested alone" ${ }^{14}$ Positive ESBL-producing isolates were identified using API 20E strips (bioMérieux, France) and stored at $-70^{\circ} \mathrm{C}$ in $\mathrm{BHI}$ broth with $15 \%$ glycerol for further molecular analysis.

\section{Molecular analysis}

All ESBL producing isolates were screened for the resistance gene CTX-M by PCR assay using primers and conditions previously described. ${ }^{15}$ Bacterial DNA extraction was performed by the boiling lysis method. ${ }^{16}$

\section{Statistical analysis}

The prevalence was calculated as the percentage of positive ESBL-E samples among all samples of each group. To assess the possible statistical associations between gender and age group fecal carriers, Fisher's exact test was used and the significance was established at $\mathrm{p}<0.05$.

\section{Results}

Seven different putative reservoirs (Human and dog feces, tap water, beef and chicken, salsas/vegetables from street vendors, and domestic flies) of ESBL-E were studied for the presence of these microorganisms. All reservoirs, except for tap water, were found to harbor ESBL-E. Table 1 shows the prevalence of these organisms in the different reservoirs included in this study.

Table I Prevalence of ESBL-E strains among different reservoirs and of CTX-M positive strains

\begin{tabular}{lll}
\hline Reservoir & ESBL-E Prevalence & CTX-M Prevalence \\
\hline All reservoirs & $48.4 \%(156 / 322)$ & $96.2 \%(150 / 156)$ \\
Human fecal carriage & $75.0 \%(78 / 104)$ & $98.7 \%(77 / 78)$ \\
Beef & $68.8 \%(22 / 32)$ & $95.5 \%(21 / 22)$ \\
Chicken meat & $66.7 \%(12 / 18)$ & $100 \%(12 / 12)$ \\
Dog fecal carriage & $53.3 \%(16 / 30)$ & $93.8 \%(15 / 16)$ \\
Fly pools & $33.3 \%(8 / 24)$ & $75.0 \%(6 / 8)$ \\
Salsas & $28.6(12 / 42)$ & $100.0 \%(12 / 12)$ \\
Vegetables & $19.0 \%(8 / 42)$ & $87.5 \%(7 / 8)$ \\
Tap water & $0.0 \%(0 / 30)$ & $0.0 \%(0 / 0)$ \\
\hline
\end{tabular}

Human participant age groups and carriage prevalence are shown in Table 2. The prevalence of fecal carriage was not significant between genders and age groups $(p>0.05)$.

One hundred and fifty six strains of ESBL-producing Enterobacteria were isolated in this study, with E. coli being, by far, the most prevalent one, which was responsible for $93.6 \%(146 / 156)$ of the total ESBL-E isolates, followed by K. oxytoca $3.8 \%(6 / 156)$ and $K$. pneumoniae, $2.6 \%(4 / 156)$. E. coli was isolated from all types of reservoirs; while $K$. oxytoca from salsas/vegetables ${ }^{4}$ and human feces ${ }^{2}$ and $K$. pneumoniae from salsas/vegetables. ${ }^{4}$ 
Table 2 Human fecal carriage among genders and age groups

\begin{tabular}{llll}
\hline Age group & $\begin{array}{l}\text { Carriage of } \\
\text { ESBL-E in women }\end{array}$ & $\begin{array}{l}\text { Carriage of } \\
\text { ESBL-E in men }\end{array}$ & $\boldsymbol{p}$ \\
\hline All ages & $69.4 \%(43 / 62)$ & $59.5 \%(25 / 42)$ & 0.401 \\
$18-33$ & $70.0 \%(21 / 30)$ & $59.1 \%(13 / 22)$ & 0.557 \\
$34-49$ & $64.7 \%(11 / 17)$ & $57.1 \%(8 / 14)$ & 0.724 \\
$>50$ & $73.3 \%(11 / 15)$ & $66.7 \%(4 / 6)$ & 1 \\
\hline
\end{tabular}

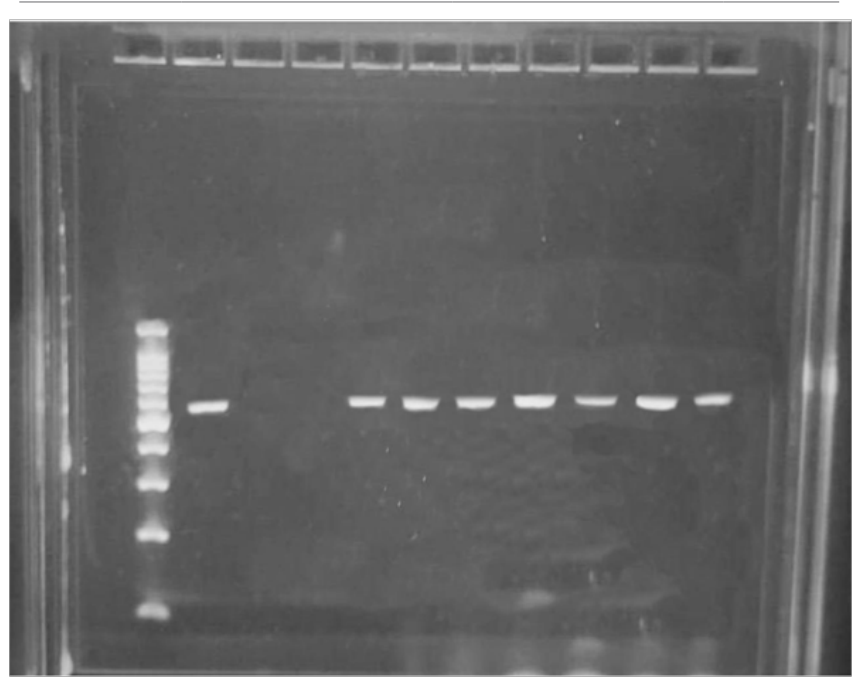

Figure I Electrophoresis of PCR products on a I\% agarose gel. Lane I, ladder I,000 bp; Lane 2, positive control (ESBL-producing E. coli previously analyzed); Lane 3, negative control (E. coli ATCC 25922); Lane 4, negative sample; Lanes 5-10, positive samples with a 590 pb PCR product.

Among all ESBL-producing strains, $96.2 \%(150 / 156)$ carried the $b l a_{\text {СтX-M }}$ gene, while only 6 of them did not $(3.8 \%)$, suggesting the presence of less common genes such as $b l a_{\mathrm{HSV}}$ and $b l a_{\mathrm{TEM}}$ genes (Figure 1). Occurrence of bla $a_{\text {СТХ-M }}$ strains among different reservoirs is shown in Table 1. All isolated Klebisella species (10/10) harbored the $b l a_{\text {СТХ-м }}$, whereas $95.9 \%(140 / 146)$ of $E$. coli were bla ${ }_{\text {СТХ-м }}$ carriers.

\section{Discussion}

Antibiotic resistance is one of the most vital threats to the public's health. The antibiotic resistance crisis has been attributed to different causes; among them are the overuse and misuse of these medications, by both clinicians and patients, extensive use in livestock and poor hygiene. ${ }^{17,18}$ Of all the known mechanisms of antibiotic resistance in Gram negative bacteria, the production of extended spectrum $\beta$-lactamases has gained great interest in the medical community worldwide, as the therapeutic options for these organisms are limited. Furthermore, the emergence and spread of ESBL-E containing the CTX-M gene is one of the most significant epidemiologic changes in infectious diseases during recent years, ${ }^{19}$ as CTX-M positive clones are currently the most frequently isolated strains as cause of community acquired infections. ${ }^{15,19-23}$

Several studies worldwide have demonstrated that ESBL-E can be transmitted from different niches to humans: $;^{21-23}$ however, to our knowledge, very few studies have been published on the reservoirs of these organisms in Mexico. As the prevalence of these bacteria in different reservoirs varies from region to region, the importance of this work lies in the fact that before implementing actions aimed at reducing their transmission, it is of extreme importance to understand the full epidemiology, including possible reservoirs, of this phenomenon.

As human fecal colonization plays a key role in the pathogenesis of ESBL-E infections, ${ }^{11}$ the first goal of our study was to determine the fecal carriage among our human population studied. Previous exposure to antibiotics has been shown to be a risk factor for the fecal colonization of these agents. As certain infections, such as those in the urinary tract, are related to the patient's own fecal microbiota, ${ }^{7,8}$ antibiotic treatment, whether correctly or incorrectly prescribed, would confer ESBL-E colonized individuals under such therapy, additional risk of infection with the patient's own fecal microbiota. Contrary to other reports in the literature, in our population there is high carriage prevalence $(75.0 \%)$ despite no previous use of antibiotics nor of hospital exposure for three months. These results indicate that additional factors could influence human colonization in our community; as different studies have shown that fecal carriage is higher in developing countries, ${ }^{23}$ one of these factors may be related to poor hygiene, including consumption of ESBL-E contaminated food.

We have long known that bacteria present in food can reach the human gut; thus our next goal in the study was to determine if food can act as reservoir of ESBL-E. Eating "antojitos" (cravings) from street vendors is a common tradition in all parts of Mexico. Unfortunately, the preparation of this type of food is not always done under the proper sanitary conditions, putting food under risk of fecal contamination, including that with ESBL-producing bacteria. As our results show, salsas/vegetables from these vendors show a high percentage $(23.8 \%)$ of positivity to ESBL-E contamination, strongly suggesting that ingestion of these foods can serve as a vehicle to reach the human gut and start colonization. To test if other foods and tap water used to wash fruits and vegetables could serve as additional vehicles of ESBL-E transmission, this type of samples were included in the present study. Our results show that tap water in our community does not contain ESBL-E, indicating that the chlorination process of municipal water inactivates these type of microorganisms and is not the vehicle by which vegetables from street vendors get contaminated. When uncooked beef and chicken meat were analyzed, both types of meat showed extremely high ESBL-E contamination rates (68.8 and $66.7 \%$, respectively), which probably takes place during the slaughtering process of the animals, that indicates lack of hygiene measures when performing such activity. The high fecal carriage rate of food animals reported worldwide is most likely caused by the selective force of antibiotic usage in these animals, either as feed additives or therapeutics.

Solà-Ginés et al. ${ }^{24}$ and others had previously shown that domestic flies (Musca domestica) can act as a vector for ESBL-E; however these studies were performed on flies with close proximity to animal manure, thus it is not surprising that they were contaminated with fecal microorganisms, including ESBL-E. The flies collected for the present study were caught in close proximity to human food, as they were caught in restaurants and household kitchens. Some studies have suggested that these arthropods can play important roles in the transmission of antibiotic resistant bacteria, acting as carriers ${ }^{25}$ the results of the present study show that flies are, indeed, vectors for such microorganisms, and due to their close proximity to human food, could easily transmit them from animal to humans and from humans to humans. The presence of ESBL-E in flies was not surprising, but the high carriage positivity of the fly pools analyzed was (33.3\%), as this 
prevalence is higher than those obtained from others, ${ }^{24,25}$ suggesting, once again, that poor hygiene may play a key role in the transmission of these agents.

A final putative reservoir of ESBL-E in our community that was investigated was companion dogs. As with other possible reservoirs included in this study, companion dogs also showed a high degree of fecal colonization, as $53.3 \%$ of animals studied showed carriage of these organisms. The close proximity between humans and dogs would allow the transmission between people and animals in the same environment, hypothesis that has been partially confirmed by others when strains isolated from humans and companion dogs have shown clonal relationships among them. ${ }^{26}$

One of the main causes in the increase of number of ESBLE-associated infections caused appears to be the increase in the propagation of the bla $a_{\text {Стх-м }}$ genes, phenomenon which started in the mid-1990s and is currently known as the CTX-M pandemic. Currently, a large number of infections worldwide, particularly those acquired in the community, are caused by strains carrying this gene, particularly the CTX-M-15 subtype. Similarly to other studies, the great majority (96.2\% of all isolates, $100 \%$ of Klebisella $s p$ and $95.9 \%$ of E. coli strains) of our ESBL-E isolates harbored $b l a_{\mathrm{CTX}-\mathrm{M}}$, confirming that these genes have spread in our region, as have in other parts of the world. No other ESBL-E carrying a bla gene shows the ability to transmit its antibiotic resistance determinant to other bacteria as efficiently as those harboring $C T X-M$; additionally, no other ESBL-E clones show the ease to contaminate/colonize different reservoirs and be transmitted so efficiently to humans, as CTX-M clones. This great adaptive success of the $b l a_{\text {СTX-M }}$-producing bacteria has been suggested by Cantón et al., ${ }^{27}$ to be driven by the presence of specific sequence types grouped in clonal complexes linked to CTX-M enzymes and to their association with resistance to other antibiotic types.

One of the major limitations of the present study is the sample size; however, even with our limited information, we can conclude that ESBL-E is present in a large number of reservoirs in our community, and their prevalence among these reservoirs is quite large. Due to the prevailing hygiene conditions and culinary traditions of some developing countries, it would not be surprising if results similar to ours are obtained in these regions in other parts of the world. A high prevalence of ESBL-E in the environment, specifically in those reservoirs that can act as vehicles for fecal colonization, could put individuals in a higher risk of developing ESBL-E associated infections, especially of infections such as UTI, that are caused by own members of the patient's fecal microbiota. However, to confirm our results, a larger scale survey is necessary to accurately evaluate the putative reservoirs of ESBL-E.

In conclusion, we have shown that bacteria producers of extended spectrum $\beta$-lactamases present a large number of reservoirs in our community and that all niches harboring these organisms presented high prevalence of positivity to ESBL-E, situations that could easily be the rule in other parts of the developing world. It has been largely accepted that overuse of antibiotics, and their selective force on bacteria, plays a key role in the appearance of antibacterial resistance clones. However, as suggested by the results of our study, lack of hygiene may play a pivotal role in the spread of ESBL-E to humans; so, in order to properly tackle the current crisis of antibiotic resistance, strict measures to improve hygiene conditions worldwide should be included.

\section{Acknowledgments}

I thank Todd Michael Pitts for his contributions to this study. This research did not receive any specific grant from funding agencies in the public, commercial, or not-for-profit sectors.

\section{Conflicts of interest}

Author declares that there is no conflicts of interest.

\section{References}

1. Philippon A, Labia R, Jacoby G. Extended-spectrum beta-lactamases. Antimicrob Agents Chemother. 1989;33(8):1131-1136.

2. Morfín-Otero R, Mendoza-Olazarán S, Silva-Sánchez J, et al. Characterization of Enterobacteriaceae isolates obtained from tertiary care hospital in Mexico, which produces extended-spectrum ß-lactamase. Microb Drug Resist. 2013;19(5):378-383.

3. Navarro-Navarro M, Robles-Zepeda RE, Garibay-Escobar A, et al. Escherichia coli y Klebsiella pneumoniae comunitarias y hospitalarias productoras de $\beta$-lactamasas en hospitales de Hermosillo, Sonora. Salud Publica Mex. 2011;53(4):341-344.

4. Apisarnthanarak A, Kiratisin P, Mundy LM. Clinical and molecular epidemiology of healthcare-associated infections due to extendedspectrum beta-lactamase (ESBL)-producing strains of Escherichia coli and Klebsiella pneumoniae that harbor multiple ESBL genes. Infect Control Hosp Epidemiol. 2008;29(11):1026-1034.

5. Leistner R, Schröder C, Geffers C, et al. Regional distribution of nosocomial infections due to ESBL-positive Enterobacteriaceae in Germany: data from the German National Reference Center for the Surveillance of Nosocomial Infections (KISS). Clin Microbiol Infect. 2015;21(3):e1-5.

6. Deitch E. Gut-Origin sepsis: evolution of a concept. Surgeon. 2012;10(6):350-356.

7. Yamamoto S, Tsukamoto T, Terai A, et al. Genetic evidence supporting the fecal-perineal-urethral hypothesis in cystitis caused by Escherichia coli. J Urol. 1997;157(3):1127-1129.

8. Ruppé E, Lixandru B, Cojocaru R, et al. Relative Fecal Abundance of Extended-Spectrum- $\beta$-Lactamase-Producing Escherichia coli Strains and Their Occurrence in Urinary Tract Infections in Women. Antimicrob Agents Chemother. 2013;57(9):4512-4517.

9. Neupane S, Dutt Pan N, Khatiwada S, et al. Correlation between biofilm formation and resistance toward different commonly used antibiotics along with extended spectrum beta lactamase production in uropathogenic Escherichia coli isolated from the patients suspected of urinary tract infections visiting Shree Birendra Hospital, Chhauni, Kathmandu, Nepal. Antimicrob Resist Infect Control. 2016;5:5.

10. Soto SM. Importance of biofilms in urinary tract infections: new therapeutic approaches. Adv Biol. 2014;543974:1-13.

11. Lo WU, Ho PL, Chow KH, et al. Fecal carriage of CTXM type extendedspectrum beta-lactamase-producing organisms by children and their household contacts. J Infect. 2010;60(4):286-292.

12. Hordijk J, Schoormans A, Kwakernaak M, et al. High prevalence of fecal carriage of extended spectrum $\beta$-lactamase/AmpC-producing Enterobacteriaceae in cats and dogs. Front Microbiol. 2013;4:242.

13. Haque A, Yoshizumi A, Saga T, et al. ESBL-producing Enterobacteriaceae in environmental water in Dhaka, Bangladesh. $J$ Infect Chem. 2014;20(11):735-737.

14. M100-S25: Performance Standards for Antimicrobial Disk Susceptibility Testing; Twenty-Fifth Informational Supplement. USA: Clinical and Laboratory Standards Institute; 2017. 240 p. 
15. Yazdi M, Nazemi A, Mirinargasi M, et al. Genotypic versus Phenotypic methods to detect Extended-Spectrum Beta - Lactamases (ESBLS) in Uropathogenic Escherichia coli. Annals of Biological Research. 2012;3(5):2454-2458.

16. De Medici D, Croci L, Delibato E, et al. Evaluation of DNA extraction methods for use in combination with SYBR green I real-time PCR to detect Salmonella enterica serotype Enteritidis in poultry. Appl Environ Microbiol. 2003;69(6):3456-3461.

17. Antibiotic resistance threats in the United States. USA: Centers for Disease Control and Prevention; 2013. 114 p.

18. Golkar Z, Bagasra O, Pace DG. Bacteriophage therapy: a potential solution for the antibiotic resistance crisis. J Infect Dev Ctries. 2014;8(2):129-136.

19. Rodriguez-Baño J, Pascual A. Clinical significance of extendedspectrum beta-lactamases. Expert Rev Anti Infect Ther. 2008;6(5):671683.

20. Njage PM, Buys EM. Quantitative assessment of human exposure to extended spectrum and AmpC $\beta$-lactamases bearing E. coli in lettuce attributable to irrigation water and subsequent horizontal gene transfer. Int J Food Microbiol. 2017;240:141-151.

21. Casella T, Nogueira MCL, Saras E, et al. High prevalence of ESBLs in retail chicken meat despite reduced use of antimicrobials in chicken production, France. Int J Food Microbiol. 2017;257:271-275.
22. Ljungquist $\mathrm{O}$, Ljungquist D, Myrenås M, et al. Evidence of household transfer of ESBL-/pAmpC-producing Enterobacteriaceae between humans and dogs-a pilot study. Infect Ecol Epidemiol. 2016;6:31514.

23. Woerther PL, Burdet C, Chachaty E, et al. Trends in Human Fecal Carriage of Extended-Spectrum $\beta$-Lactamases in the Community: Toward the Globalization of CTX-M. Clin Microbiol Rev. 2013;26(4):744-758.

24. Solà-Ginés M, González-López JJ, Cameron-Veas K, et al. Houseflies (Musca domestica) as Vectors for Extended-Spectrum $\beta$-LactamaseProducing Escherichia coli on Spanish Broiler Farms. Appl Environ Microbiol. 2015;81(11):3604-3611.

25. Liu Y, Yang Y, Zhao F, et al. Multi-drug resistant gram-negative enteric bacteria isolated from flies at Chengdu Airport, China. Southeast Asian J Trop Med Public Health. 2013;44(6):988-996.

26. Carvalho AC, Barbosa AV, Arais LR, et al. Resistance patterns, ESBL genes, and genetic relatedness of Escherichia coli from dogs and owners. Braz J Microbiol. 2016;47(1):150-158.

27. Cantón R, González-Alba JM, Galán JC. CTX-M Enzymes: Origin and Diffusion. Front Microbiol. 2012;3:110. 